\title{
Acute administration of methylphenidate alters the prefrontal cortex neuronal activity in a dose-response characteristic
}

This article was published in the following Dove Press journal:

Journal of Experimental Pharmacology

II February 2014

Number of times this article has been viewed

\section{Catherine M Claussen Nachum Dafny \\ Department of Neurobiology and Anatomy, University of Texas Health Science Center Medical School at Houston, Houston, TX, USA}

\begin{abstract}
The prefrontal cortex (PFC) is part of the collective structures known as the motive circuit. The PFC acts to enhance higher cognitive functions as well as mediate the effects of psychostimulants. Previous literature shows the importance of PFC neuronal adaptation in response to acute and chronic psychostimulant exposure. The PFC receives input from other motive circuit structures, including the ventral tegmental area, which mediates and facilitates the rewarding effects of psychostimulant exposure. PFC neuronal and locomotor activity from freely behaving rats previously implanted with permanent semimicroelectrodes were recorded concomitantly using a telemetric (wireless) recording system. Methylphenidate (MPD) is used as a leading treatment for behavioral disorders and more recently as a cognitive enhancer. Therefore, the property of MPD dose response on PFC neuronal activity was investigated. The results indicate that MPD modulates PFC neuronal activity and behavioral activity in a dose-dependent manner. PFC neuronal responses to $0.6 \mathrm{mg} / \mathrm{kg}$ elicited mainly a decrease in PFC neuronal activity, while higher MPD doses $(2.5$ and $10.0 \mathrm{mg} / \mathrm{kg}$ ) elicited mainly increased neuronal activity in response to MPD. The correlation between MPD effects on PFC neuronal activity and animal behavior is discussed.
\end{abstract}

Keywords: prefrontal cortex, Ritalin, behavior, neuronal, acute

\section{Introduction}

Methylphenidate (MPD) enhances cognitive and behavioral function by acting, at least in part, on the prefrontal cortex (PFC). Recent reports state that the use of MPD as a cognitive enhancer has become widely abused among college students and adults. ${ }^{1-3}$ Due to the PFC's critical role in higher cognitive functions, it is considered one of the main brain regions to respond to acute and chronic MPD administration. ${ }^{4,5}$ MPD binds to dopamine (DA) and noradrenaline (NE) transporters, blocking the reuptake of both DA and NE from the synaptic cleft to the presynaptic terminals, therefore leading to an increased level of DA and NE in the extracellular space. ${ }^{6-8}$

The PFC is one of multiple structures that comprises the motive circuit; other structures include the nucleus accumbens and ventral tegmental area (VTA). The motive circuit drives reward and underlies psychostimulant actions on the brain. ${ }^{6}$ The PFC receives DA input from the VTA and descends glutamatergic transmission to the VTA to facilitate the rewarding effects of psychostimulants. A cascade of neural events following repetitive MPD exposure can lead to the augmented behavioral response of behavioral sensitization. ${ }^{9,10}$ Behavioral sensitization is characterized by an increase in the behavioral response to repeated psychostimulant exposure. ${ }^{11}$ The ability of a psychostimulant to elicit behavioral sensitization is predominately facilitated by both $\mathrm{D}_{1}$ and $\mathrm{D}_{2}$ DA receptors in 
the motive circuit ${ }^{12}$ and $\alpha 2$ adrenoceptors of the PFC. ${ }^{6}$ It was reported that low doses of MPD improve working memory by way of strengthening the $\alpha 2$-adrenoceptors and $\mathrm{D}_{1} \mathrm{DA}$ transmission in the PFC, ${ }^{6}$ whereas higher doses led to behavioral sensitization via a robust increase in DA release in the PFC and other motive-circuit structures. ${ }^{13}$ Although higher doses of MPD may lead to excessive stimulation of the $\mathrm{D}_{1}, \alpha_{1}$, and/or $\beta_{1}$ receptors, ${ }^{13}$ its explicit receptor mechanism underlying side effects like addiction and other physiological effects remains mostly unexplored.

The aim of this experiment was to elucidate whether escalating doses of acute MPD $(0.6,2.5$, and $10.0 \mathrm{mg} / \mathrm{kg})$ lead to electrophysiological differences in PFC neuronal responses. Therefore, this study recorded concomitantly from animals previously implanted with four electrodes in the PFC both freely behaving locomotion and neuronal activity using a wireless (telemetric) device attached to the headstage to allow a freer range of motion in response to MPD.

\section{Methods}

\section{Subjects}

Twenty-six adult male Sprague Dawley rats were received from Harlan Laboratories (Indianapolis, IN, USA). Animals were placed individually in Plexiglas cages for 1 week for adaptation. The room was maintained at an ambient temperature of $21^{\circ} \mathrm{C} \pm 2{ }^{\circ} \mathrm{C}$ and at a humidity of $58 \%-62 \%$ with a 12 -hour light/ dark cycle. Rats were supplied food and water ad libitum for the entire duration of the study. All experiments were approved by our Animal Welfare Committee and carried out in accordance with the National Institute of Health's Guide for the Care and Use of Laboratory Animals. To eliminate environmental contribution to the drug effect, such as a novel condition, the injections and recordings were done in the animal's home cage.

\section{Drug}

MPD was obtained from Mallinckrodt Pharmaceuticals (St Louis, MO, USA). Based on previous dose-response experiments (from 0.1 to $40 \mathrm{mg} / \mathrm{kg}$ intraperitoneal MPD), doses of $0.6,2.5$, and $10.0 \mathrm{mg} / \mathrm{kg}$ MPD administered intraperitoneally in the morning were selected, since these doses elicited behavioral sensitization or tolerance. ${ }^{14-21}$ MPD was dissolved in $0.9 \%$ saline $(\mathrm{NaCI})$ solution, and the dose was calculated as free base. All injections were equalized to $0.8 \mathrm{~mL}$ with saline and were administered between 8 am and 9 am.

\section{Surgery}

On the day of surgery, rats were weighed and anesthetized with $2 \%-4 \%$ isoflurane. The top of the rats' heads were shaved to expose the skin and coated with a thin layer of $2 \%$ lidocaine hydrochloride jelly (Akorn Inc., Lake Forest, IL, USA). Once the animals were placed into the stereotaxic instrument, a 1-inch incision was made, removing the muscle and connective tissue to expose the skull. A hole was made above the frontal sinus for the reference electrode, and two bilateral holes $0.6 \mathrm{~mm}$ in diameter were drilled over the PFC in accordance with the coordinates derived from Paxinos and Watson ${ }^{22}$ (3.0 mm anterior from bregma, $0.6 \mathrm{~mm}$ lateral from midline). Six anchor screws were used in vacant areas to secure the skullcap to the skull with dental acrylic. Two twisted nickel-chromium, diamel-coated wire electrodes (fully insulated except at tips), 60 microns in diameter (70-80 M $\Omega$ ), were each secured to a $1 \mathrm{~cm}$ copper connector pin made prior to surgery. These electrodes record extracellular activity of $0.1-0.3 \mathrm{mV}$ in amplitude and 0.8-1.2 milliseconds' spike width. The reference electrode was placed in the frontal sinus, and two twisted recording electrodes were implanted in each PFC as follows: one twisted electrode (ie, two electrodes together) was inserted into the drilled hole at an initial depth of $3.0 \mathrm{~mm}$. Spiking activity was monitored during placement of electrodes by using a Grass Hi Z probe connected to a Grass P511 series preamplifier (Grass Technologies, Warwick, RI, USA). A signal-to-noise ratio of 3:1 spike to noise was used as the ratio criteria to fix the electrodes to the skull. When the activity was not sufficient, the electrode was adjusted downward in approximate increments of 10 microns until the sufficient (3:1) noise ratio of neuronal activity was observed. Upon appropriate electrode signal, cyanoacrylate surgical adhesive (Webglue ${ }^{\mathrm{TM}}$; Patterson Veterinary, Devens, MA, USA) was used to fix the electrode in the skull. Identical procedures were carried out for the secondary twisted electrode implanted in the other hemisphere. ${ }^{18,19,23-28}$ Amphenol plugs were positioned on the skull after the electrode connector pins were inserted, and then secured to the skull with dental acrylic cement. Recovery from surgical procedures lasted approximately 4-7 days. After recovery, every day animals were placed in the experimental behavioral apparatus for 2 hours and connected to the wireless (telemetric) headstage transmitter (Triangle BioSystems, Durham, NC, USA) for daily acclimation to the recording.

\section{Experimental protocol}

Animals were randomly assigned into four groups: saline, 0.6, 2.5, or $10.0 \mathrm{mg} / \mathrm{kg}$ MPD. Experimentation began 4-7 days postsurgery, when animals were approximately 60-62 days old. On the day of experimentation, prior to the start of the recording session, animals were again allowed to acclimate 
to the recording system for 1 hour. During acclimation, the recording parameters were organized in order to properly record, analyze, and save PFC neuronal activity. All experimental groups started with a single saline $(0.8 \mathrm{~mL}$ of $0.9 \%$ ) injection, with a 60 -minute baseline of neuronal and behavioral activity recorded simultaneously. Next, a saline, $0.6,2.5$, or $10.0 \mathrm{mg} / \mathrm{kg}$ MPD injection was administered, with recordings resumed for another 60 minutes. The salineinjection group was used as a control for handling, injection, and injection volume. ${ }^{18-20,27-30}$

\section{Behavioral apparatus}

Locomotor activity was recorded using an open-field computerized animal-activity system (Opto-M3; Columbus Instruments, Columbus, OH, USA). The animals were housed in clear acrylic cages that fit into the recording apparatus, thus allowing us to record the animal's behavior in their home cages. The Columbus open-field system comprises infrared beam sensors that run $40 \mathrm{~cm}$ in length and $20 \mathrm{~cm}$ in width, with $16 \times 8$ infrared beams respectively, and their sensors set $5 \mathrm{~cm}$ above the floor of the cage. Movement across any of the infrared beams would result in a beam break, and was subsequently recorded. The breaking beams and their time were counted and compiled and downloaded to a PC in 10-minute bin increments and were evaluated from 60 minutes postinjection for both the baseline and MPD administration.

\section{Analysis of behavioral data}

The Opto M3 software summed each broken beam into 10-minute bins for 60 minutes (ie, six bins/hour) and calculated the horizontal activity (HA) and the number of stereotypy activities (NOS). An analysis of variance was used on the HA to determine if as a group the animals exhibited significant $(P<0.05)$ change in their locomotor activity following MPD when compared to the locomotion recorded during the baseline control activity. HA records overall locomotor activity and NOS, which counts the number of repetitivemovement episodes with at least a 1-second interval before the beginning of another episode of movements.

\section{Electrophysiological recording Data acquisition}

Rats were placed in Faraday testing boxes, inside their home cages, during the experimental recording day to reduce noise during signal transmission. The wireless headstage (weighing less than $5 \mathrm{~g}$ ) was connected to the electrode pins of the skull cap. The headstage sent neuronal activity signals through a receiver that connected to a Cambridge Electronic
Design (CED) analog-to-digital converter (Micro1401-3; CED, Cambridge, England) that was connected to a $\mathrm{PC}$ that then collected and stored the recorded data on a PC. Spike 2.7 software (CED) was used offline to sort neuronal activity for identical spike amplitude and waveforms by examining single-unit spike activity exhibiting similar amplitude and waveform patterns before and after MPD administration (see the following "Spike sorting" section for more details) to produce a sequential frequency histogram and to calculate the firing rate in spikes per second. Approximately one to two spikes (units) were analyzed per electrode.

\section{Spike sorting}

Recording with sampling rates up to $200 \mathrm{kHz}$ (analog) was captured by the program and processed using low- and highpass filters $(0.3-3 \mathrm{kHz})$. Spikes with peak amplitudes set by the two windows (positive-going and negative-going) were used to create templates. Spikes were defined using one 1,000 waveform data points. Spikes that entered into the defined amplitude window were extracted, and peak amplitudes outside this window were rejected. This algorithm allowed the creation of templates that provided high-dimensional reference points that were used to perform accurate spike sorting. Furthermore, this algorithm controlled for the influence of noise, spurious threshold crossing, and waveform overlap. All temporally displaced templates were compared with the selected spike event to find the best-fitting template that yielded the minimum residue variance. Secondly, a template-matching procedure was then performed; when the distance between the template and waveform exceeded a threshold (80\%), the waveforms were rejected. That meant that the spike-sorting accuracy in the reconstructed data was about $95 \%$.

\section{Analysis of the electrophysiological data}

The sorted neuronal activity obtained from the fixed-template matching system was converted by software into firing rates (spikes per second) for the baseline control recording and for the 60-minute activity following MPD administration. The generated firing rates were entered into a spreadsheet to display the rat's number, experimental day, baseline activity, activity of each post-MPD dose, and channel (to distinguish hemisphere).

Due to the firing rates not holding to normality assumptions, we used the critical ratio (CR) test to assess the effect of the drug on the baseline activity. This test was used to determine whether acute MPD treatments altered the PFC unit activity $(\mathrm{CR}=\mathrm{R}-\mathrm{C} / \sqrt{\mathrm{R}}+\mathrm{C} \pm 1.96=P<0.05)$, where 
$\mathrm{C}$ represents the 60 -minute activity immediately following saline and $\mathrm{E}$ the 60-minute activity immediately post-MPD injection for each unit; a value of more than +1.96 indicates that the treatment elicited significant increased activity, while a value of more than -1.96 indicates that the treatment elicits significant attenuation in activity. As a second measure to verify a significant change in firing rate from baseline to drug, each unit activity following MPD exposure had to change its firing rate by at least two standard deviations from the baseline 60 -minute average. ${ }^{24,25,31,32}$ The percentage change in mean neuronal firing rates was calculated for the 60 minutes post-MPD injection compared to baseline activity for each unit activity and averaged for all three doses.

\section{Histological verification of electrode placement}

The rats were killed with sodium pentobarbital following the end of the experiment. A perfusion with $10 \%$ formalin solution containing 3\% potassium ferrocyanide was performed. A small lesion was created in the electrode location by passing a $2 \mathrm{~mA}$ current through the electrode connector pin for 40 seconds. The brain was then excised and stored in $10 \%$ formalin for subsequent histological processing. Electrode placement was verified using coronal sections (60 microns thick) and stained with cresyl violet, using a rat-brain atlas ${ }^{32}$ (Figure 1).

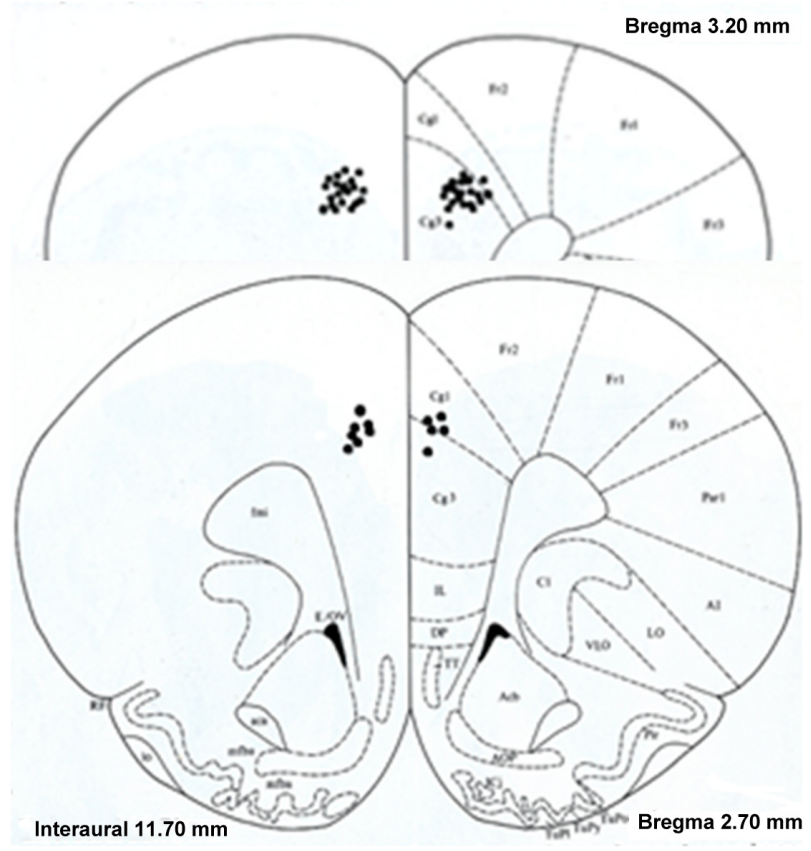

Figure I Reconstruction of the histological verification of prefrontal cortex (PFC) electrode placement. All 52 twisted electrodes were securely placed in the PFC, and the recordings from these electrodes were evaluated.

\section{Results}

\section{Locomotor behavior}

Twenty-six rats met the histological verification of electrode placement within the PFC (Figure 1) and exhibited at least a 3:1 noise-to-spike ratio amplitude in order to be included in the study (saline, $\mathrm{n}=4 ; 0.6 \mathrm{mg} / \mathrm{kg}, \mathrm{n}=7 ; 2.5 \mathrm{mg} / \mathrm{kg}, \mathrm{n}=9$; $10.0 \mathrm{mg} / \mathrm{kg}, \mathrm{n}=6$ ). The control group showed no effect on behavioral activity following saline injections. Figure 2 summarizes the behavioral data following MPD exposure for all doses $(0.6,2.5$, and $10.0 \mathrm{mg} / \mathrm{kg}$ MPD) and shows doseresponse accelerated increase in activity with increasing MPD dose. The NOS activity exhibited identical observations as the HA, and is shown in Figure 2 (upper-right corner).

\section{Electrophysiological responses}

A total of 272 PFC units were recorded from 104 electrodes (four electrodes per animal) with identical spike waveform and amplitude during the experiment. Approximately one to three units were evaluated per electrode: 40 units were recorded and evaluated from the saline group, 86 units from the $0.6 \mathrm{mg} / \mathrm{kg}$ MPD group, 84 units from animals undergoing $2.5 \mathrm{mg} / \mathrm{kg}$ MPD treatment, and 62 units from animals treated with $10.0 \mathrm{mg} / \mathrm{kg}$ MPD.

\section{PFC units exposed to saline only (control)}

Forty PFC units were recorded from four rats injected twice with saline only. In general, these PFC units exhibited similar neuronal firing activity following the second saline injection compared to the initial saline injection. Two PFC units altered their activity after the second saline injection compared to the activity observed after the first saline injection. One unit exhibited increased activity, and one exhibited decreased activity. This observation following saline injection (control) shows that handling and injection volume did not modulate the PFC unit's neuronal firing rates. Therefore, the activity following the initial saline injection was used as the baseline activity to compare to the activity following MPD exposure.

\section{Overall PFC units exposed to MPD}

Figure 3 summarizes in percentages the total number of PFC units exhibiting significant $(P<0.05)$ change in firing rates (white column), and how many of them responded significantly $(P<0.05)$ by increasing (striped column) or decreasing (black column) in their PFC neuronal firing rates following $0.6,2.5$, and $10.0 \mathrm{mg} / \mathrm{kg}$ MPD.

Exposure to $0.6,2.5$, and $10.0 \mathrm{mg} / \mathrm{kg}$ MPD altered significantly $(P<0.05)$ the firing rate of 78 of $86(91 \%)$, 


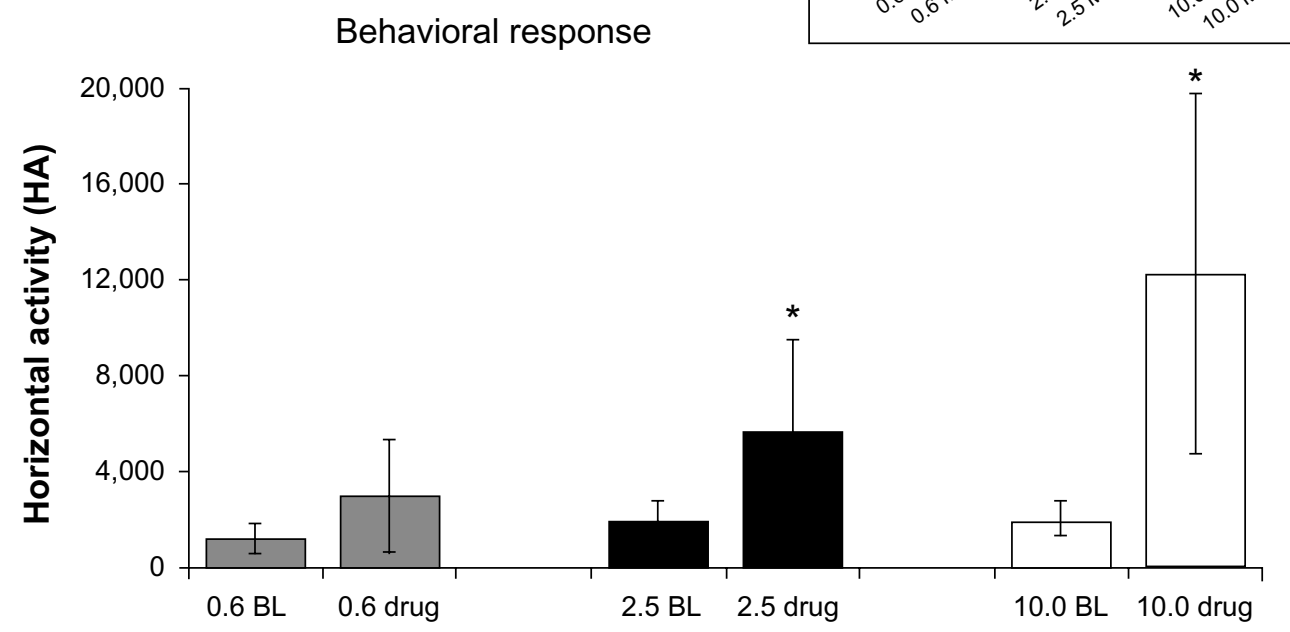

Figure 2 Summary of locomotor behavioral data. The upper-right corner shows the number of stereotypic movements (NOS) following $0.6,2.5$, and $10.0 \mathrm{mg} / \mathrm{kg}$ methylphenidate (MPD) as compared to baseline (BL) activity. Horizontal activity (HA) following $0.6,2.5$, and $10.0 \mathrm{mg} / \mathrm{kg}$ MPD was compared to BL activity. Note: $* P<0.05$.

74 of $84(88 \%)$, and 51 of $62(82 \%)$ PFC units, respectively (Figure 3, total responsiveness). From the units responding to MPD exposure, 24 of 78 (31\%), 37 of 74 (50\%), and 41 of $51(86 \%)$ expressed increased activity, while 54 of $78(69 \%)$, 37 of $74(50 \%)$, and seven of 51 (14\%) exhibited a decrease in their neuronal activity following $0.6,2.5$, and $10.0 \mathrm{mg} / \mathrm{kg}$ MPD, respectively. The $0.6 \mathrm{mg} / \mathrm{kg}$ MPD group elicited mainly a decrease in PFC neuronal activity (Figure 4A), while with increasing MPD dose, more PFC units responded to MPD by increasing their neuronal activity following $0.6,2.5$ (Figure 4B), and $10.0 \mathrm{mg} / \mathrm{kg}$ MPD (Figure 4C), respectively.

\section{Comparing the percentage change (intensity) in firing rate following MPD exposure}

Figure 5 histogram represents the percentage change in firing rate of PFC units responding to MPD exposure compared to their baseline activity (set arbitrarily as 100\%), for all doses. The PFC units that increased their neuronal firing rates (in percentage) recorded from all animals with no correlation with behavior exhibited a $21 \%, 24 \%$, and $30 \%$ increase in neuronal activity and those exhibiting significant decreases in firing rates showed a $76 \%, 16 \%$, and $68 \%$ decrease in firing

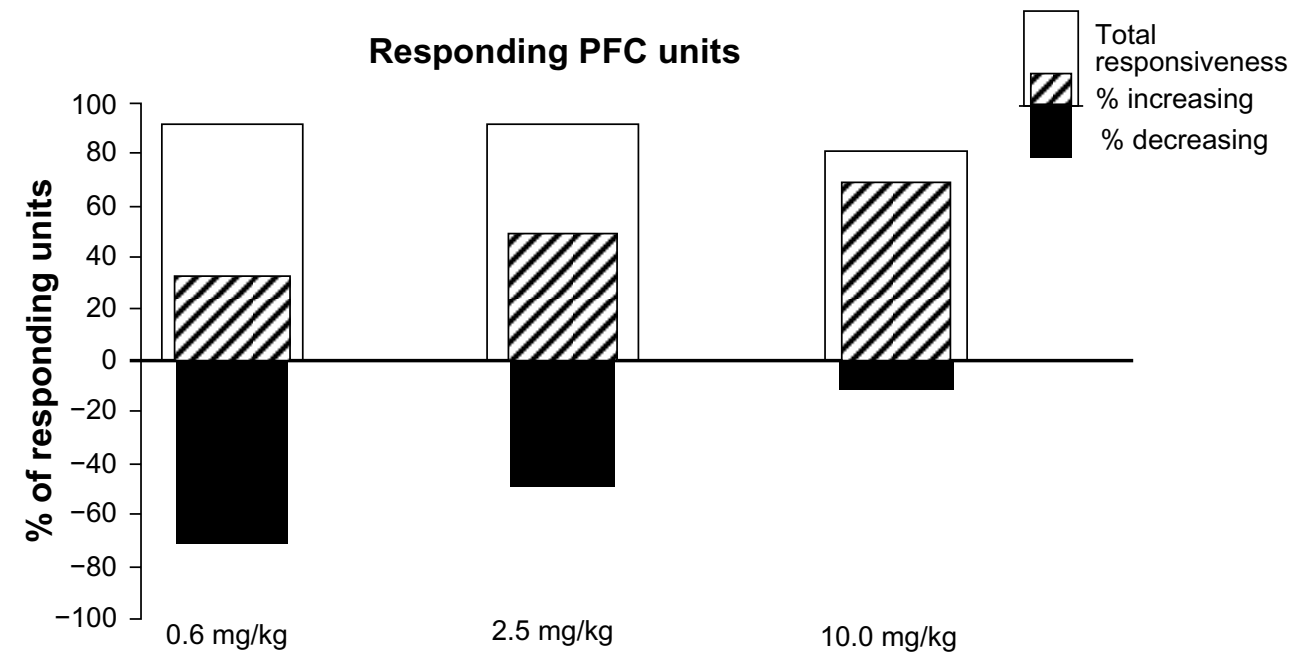

Figure 3 Histogram summarizing the percentage of the total number of units responding and the response direction in percentage that either increased or decreased following $0.6,2.5$, and $10.0 \mathrm{mg} / \mathrm{kg}$ methylphenidate.

Abbreviation: PFC, prefrontal cortex. 

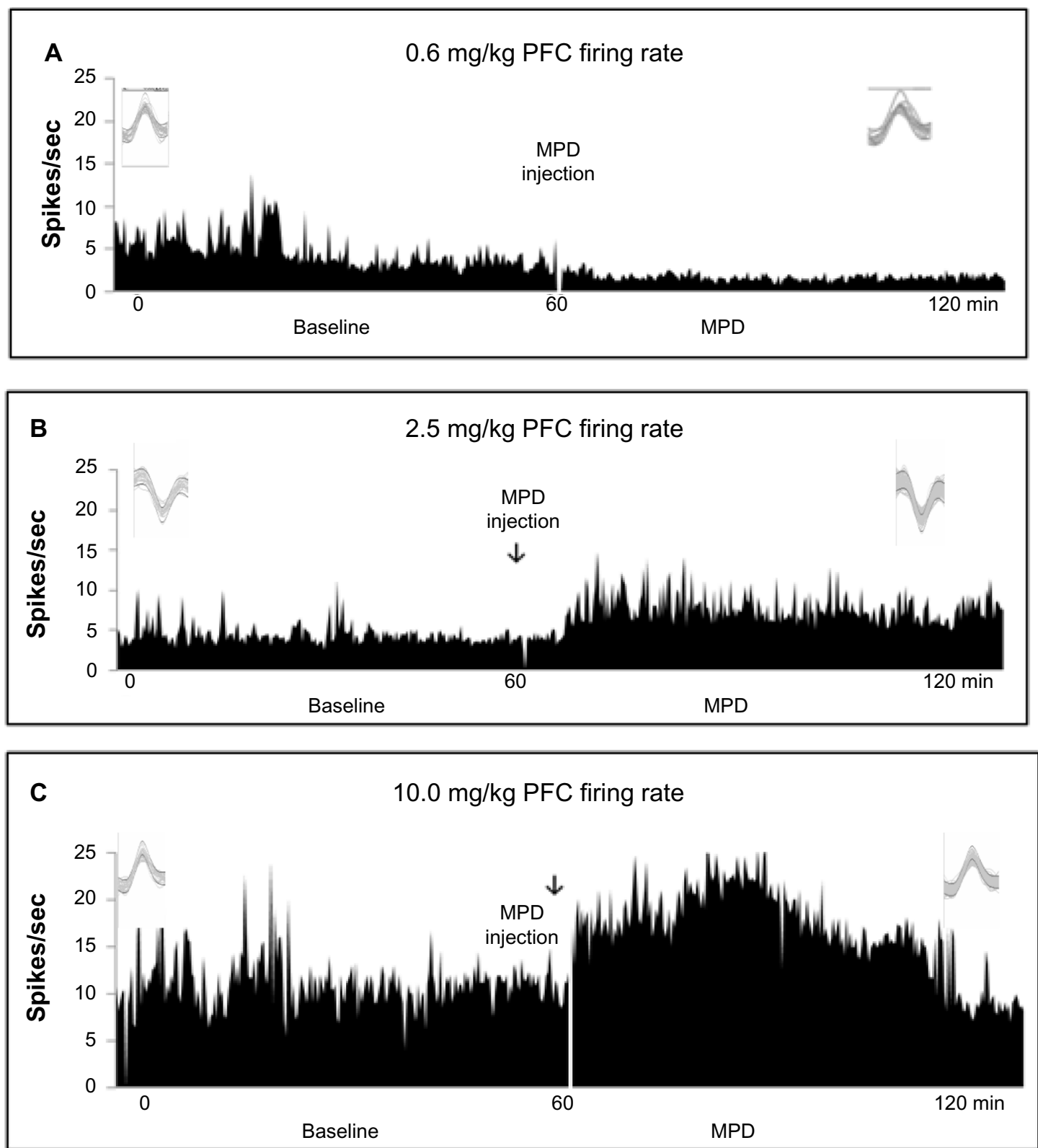

Figure 4 (A-C) Representative firing rates of prefrontal cortex (PFC) units following 0.6, 2.5, and I0.0 mg/kg methylphenidate (MPD). The frame above each histogram shows 20 superimposed spikes following saline and MPD for the same unit. (A) Unit expressing a decrease in neuronal firing following administration following $0.6 \mathrm{mg} / \mathrm{kg}$ MPD exposure. (B) Unit expressing an increase in neuronal firing following administration of $2.5 \mathrm{mg} / \mathrm{kg}$ MPD. (C) Unit expressing an increase in neuronal firing following administration of $10.0 \mathrm{mg} / \mathrm{kg}$ MPD.

following initial $0.6,2.5$, and $10.0 \mathrm{mg} / \mathrm{kg}$ MPD compared to their baseline activity, respectively.

\section{Discussion}

The steady increase in both prescription use and illicit use of MPD leads to the question of how psychostimulants work on the brain and what the behavioral and neuronal consequences of varying doses are. The PFC is a site of particular interest, because of its role in cognition, and MPD is known for its cognitive-enhancement effect. Therefore, in this study we attempted to unravel the PFC neuronal responses to low and high doses of MPD. Furthermore, we attempted to find correlations between the neuronal responses to MPD exposure and the behavioral locomotor responses to MPD.

A previously published study ${ }^{32}$ used a single dose of $2.5 \mathrm{mg} / \mathrm{kg}$ MPD and an older model of the wireless (telemetric) recording system (Alpha Omega, Nazareth, Israel) in which animal movement was constricted by a jacket holding a heavy battery pack that was worn throughout the experimental recordings. During the current experiment, a new wireless telemetric device attached (see the "Materials and methods" section) to the skull weighing less than $5 \mathrm{~g}$ 


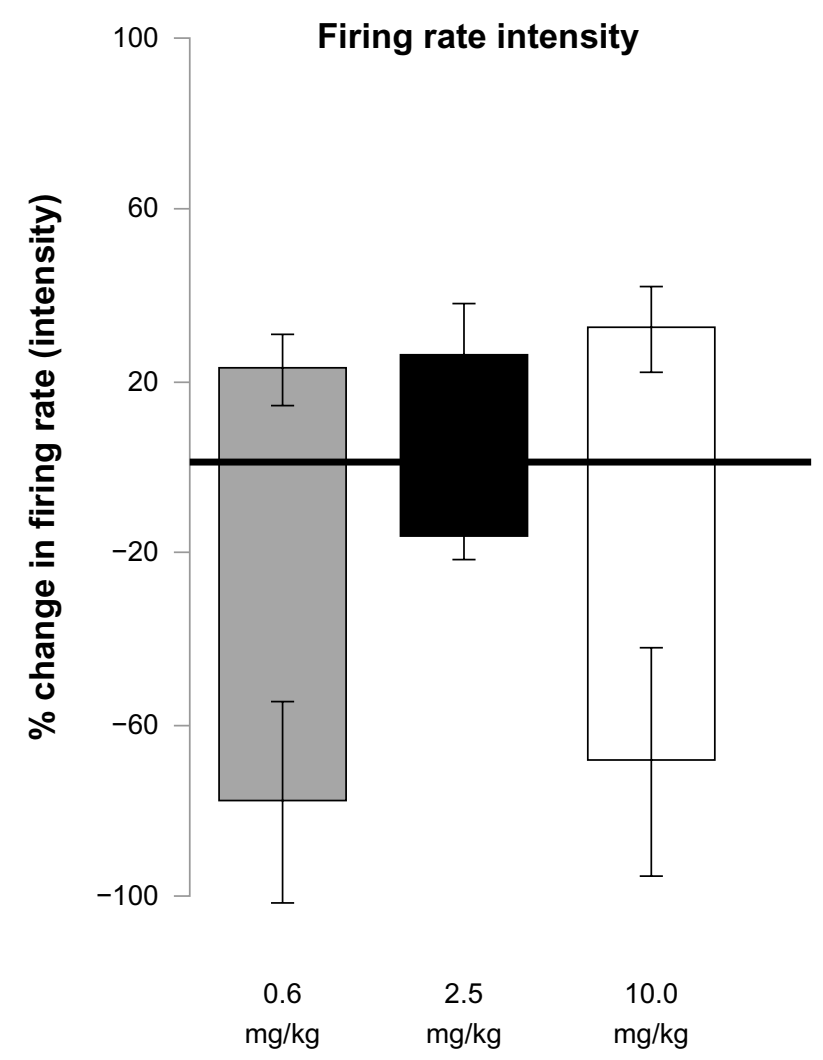

Figure 5 Summary of the intensity of the direction of the firing pattern (baseline set arbitrarily as $0 \%$ ) of prefrontal cortex units following $0.6,2.5$, and $10.0 \mathrm{mg} / \mathrm{kg}$ MPD, respectively.

was used, thus allowing full range of motion for the duration of the experiment. The results from the previous study following the $2.5 \mathrm{mg} / \mathrm{kg}$ MPD acute injection were statistically compared to the results from the present $2.5 \mathrm{mg} / \mathrm{kg}$ MPD observation. There was a significant $(P<0.001)$ difference in the response direction (increase/decrease) of PFC neurons. We posit that the difference was a result of the modulated activity due to wearing of the heavy battery pack, thus verifying our reasoning for duplicating the study using advanced technology. Furthermore, this study gives a more appropriate picture of the true PFC activity in response to acute $2.5 \mathrm{mg} / \mathrm{kg}$ MPD exposure.

The main behavioral finding of this study shows that MPD has a dose-dependent effect on locomotor activity. The $0.6 \mathrm{mg} / \mathrm{kg}$ MPD in $58 \%$ of the rats elicited a significant increase in locomotor activity, while the others showed attenuation in their locomotor response, whereas all animals exposed to 2.5 and $10.0 \mathrm{mg} / \mathrm{kg}$ MPD expressed a dosedependent characteristic of significant increases in locomotor activity. The electrophysiological recordings show an MPD dose-dependent response for those PFC units that exhibited increased neuronal activity, while those PFC units that exhibited decreases in their neuronal firing rates following MPD exposure showed a decline in the percentage responding with escalating doses.

In previous studies, ${ }^{18,19,27}$ multiple saline injections did not alter neuronal activity; therefore, the activity following saline was used as a control for handling and injection volume. The percentage of PFC neurons responding to 0.6, 2.5 , and $10.0 \mathrm{mg} / \mathrm{kg}$ MPD was similar across all the MPD doses; however, the direction of the response (increased or decreased) to each MPD dose was different. The majority of units showed a far more significant decrease in their neuronal firing rate in response to $0.6 \mathrm{mg} / \mathrm{kg}$ MPD compared to their response to $10.0 \mathrm{mg} / \mathrm{kg}$, which elicited primarily an increase in PFC neuronal firing rates.

MPD binds to the DA transporter to block the reuptake of DA into the presynaptic cleft, therefore resulting in an increase in extracellular DA levels. There are two subgroups of DA receptors: $\mathrm{D}_{1}$ and $\mathrm{D}_{2}$ receptors. ${ }^{33}$ It was reported that activation of the $\mathrm{D}_{1} \mathrm{DA}$ receptor results in an increase in neuronal firing rates, ${ }^{33,34}$ and activation of the $\mathrm{D}_{2}$ DA receptor results in an attenuation of neuronal firing activity. ${ }^{34}$ A number of in vitro intracellular studies indicate that the DA $\mathrm{D}_{1}$ and $\mathrm{D}_{2}$ receptors play a major role in controlling the excitability of PFC neurons. ${ }^{35,36}$ A previous study demonstrated in vivo that $D_{1} D A$-receptor simulation directly activates the pyramidal PFC neurons, thus reiterating the importance of DA in the MPD mechanism of action. ${ }^{34}$ Moreover, it was shown that exposure to higher MPD doses modulates the inhibitory effect of the presynaptic DA autoreceptors and causes synaptic activation that results in subsequent amplification of DA signals, thus resulting in an overall excitatory response. ${ }^{8}$ It has also been shown that the $\alpha_{2 \mathrm{~A}}$ adrenoreceptors modulate postsynaptic stimulation on pyramidal cells and work to enhance the DA receptors' signal strength. ${ }^{37}$ Using the $\alpha_{2 \mathrm{~A}}$ adrenoreceptor antagonist yohimbine to suppress the excitatory effects of MPD exposure, it is believed that $\alpha_{2 \mathrm{~A}}$-receptor activation mediates the excitability of PFC pyramidal neurons found in the PFC. $\mathrm{D}_{1}$ DA-receptor activation leads to increased excitability of pyramidal neurons, while $\alpha_{2 \mathrm{~A}}$ receptors help to inhibit overexcitability working oppositely from the $D_{1}$ receptors to tune synaptic output to a more optimal state. ${ }^{34}$

It is possible that the low dose of MPD activates prominently the $\mathrm{D}_{2}$ DA receptors, which results in firing-rate attenuation as observed in this study, whereas the higher doses of MPD activate the $\mathrm{D}_{1} \mathrm{DA}$ receptors, resulting in an increase in PFC neuronal activity and locomotion. ${ }^{38}$ Moreover $\alpha_{2 \mathrm{~A}}$ adrenoreceptors could be responsible for the inhibitory response observed at low doses, as observed 
in selective $\alpha_{2 \mathrm{~A}}$-antagonist studies in which MPD activity was suppressed. ${ }^{39}$ The PFC cytoarchitecture is highly heterogeneous; the pyramidal neurons contain DA, NE, and glutamate receptors. The $\mathrm{D}_{1} \mathrm{DA}$ receptors are expressed in a higher density compared to $\mathrm{D}_{2} \mathrm{DA}$ receptors. Therefore, it is possible to assume also from our observation that the low dose of MPD $(0.6 \mathrm{mg} / \mathrm{kg})$ activates mainly the $\mathrm{DA} \mathrm{D}_{2}$ receptors, which are involved in the therapeutic effect of attention deficit/hyperactivity disorder (ADHD), and the higher MPD dose of $2.5 \mathrm{mg} / \mathrm{kg}$ activates the $\mathrm{D}_{1} \mathrm{DA}$ receptors, which results in cognitive enhancement, while the $10.0 \mathrm{mg} / \mathrm{kg}$ MPD elicits its euphoric effects and may cause dependent responses such as behavioral sensitization or tolerance.

In conclusion, this study verifies the importance of the PFC in the pharmacotherapeutics of MPD in ADHD and its cognitive-enhancement effects.

\section{Acknowledgments}

This study was supported by an NIH DA R01 027222 grant. The authors wish to thank Zachary Jones and Dr Bin Tang for their technical support.

\section{Disclosure}

The authors report no conflicts of interest in this work.

\section{References}

1. Bidwell LC, McClernon FJ, Kollins SH. Cognitive enhancers for the treatment of ADHD. Pharmacol Biochem Behav. 2011;99:262-274.

2. Greely H, Sahakian B, Harris J, et al. Towards responsible use of cognitive-enhancing drugs by the healthy. Nature. 2008;456: $702-705$.

3. Kollins SH. Comparing the abuse potential of methylphenidate versus other stimulants: a review of available evidence and relevance to the ADHD patient. J Clin Psychiatry. 2003;64 Suppl 11:14-18.

4. Arnsten AF. Stimulants: therapeutic actions in ADHD. Neuropsychopharmacology. 2006;31:2376-2383.

5. Kieling C, Genro JP, Hutz MH, Rohde LA. A current update on ADHD pharmacogenomics. Pharmacogenomics. 2010;11:407-419.

6. Berridge CW. Neural substrates of psychostimulant-induced arousal. Neuropsychopharmacology. 2006;31:2332-2340.

7. Gatley SJ, Volkow ND, Gifford AN, et al. Dopamine-transporter occupancy after intravenous doses of cocaine and methylphenidate in mice and humans. Psychopharmacology (Berl). 1999;146:93-100.

8. Volkow ND, Wang GJ, Fowler JS, Ding YS. Imaging the effects of methylphenidate on brain dopamine: new model on its therapeutic actions for attention-deficit/hyperactivity disorder. Biol Psychiatry. 2005;57:1410-1415.

9. Kita H, Kitai ST. Amygdaloid projections to the frontal cortex and the striatum in the rat. J Comp Neurol. 1990;298:40-49.

10. Nestler EJ. Molecular mechanisms of drug addiction. Neuropharmacology. 2004;47 Suppl 1:24-32.

11. Pierce RC, Kalivas PW. Repeated cocaine modifies the mechanism by which amphetamine releases dopamine. J Neurosci. 1997;17:3254-3261.

12. Deadwyler SA, Hayashizaki S, Cheer J, Hampson RE. Reward, memory and substance abuse: functional neuronal circuits in the nucleus accumbens. Neurosci Biobehav Rev. 2004;27:703-711.
13. Arnsten AF, Dudley AG. Methylphenidate improves prefrontal cortical cognitive function through alpha2 adrenoceptor and dopamine D1 receptor actions: relevance to therapeutic effects in attention deficit hyperactivity disorder. Behav Brain Funct. 2005;1:2.

14. Askenasy EP, Taber KH, Yang PB, Dafny N. Methylphenidate (Ritalin): behavioral studies in the rat. Int J Neurosci. 2007;117:757-794.

15. Dafny N, Yang PB. The role of age, genotype, sex, and route of acute and chronic administration of methylphenidate: a review of its locomotor effects. Brain Res Bull. 2006;68:393-405.

16. Gaytan O, Ghelani D, Martin S, Swann A, Dafny N. Methylphenidate: diurnal effects on locomotor and stereotypic behavior in the rat. Brain Res. 1997;777:1-12.

17. Gaytan O, Yang P, Swann A, Dafny N. Diurnal differences in sensitization to methylphenidate. Brain Res. 2000;864:24-39.

18. Yang PB, Swann AC, Dafny N. Acute and chronic methylphenidate dose-response assessment on three adolescent male rat strains. Brain Res Bull. 2006;71:301-310.

19. Yang PB, Swann AC, Dafny N. Methylphenidate treated at the test cage - dose-dependent sensitization or tolerance depend on the behavioral assay used. Crit Rev Neurobiol. 2007;19:59-77.

20. Yang PB, Swann AC, Dafny N. Psychostimulants given in adolescence modulate their effects in adulthood using the open field and the wheelrunning assays. Brain Res Bull. 2010;82:208-217.

21. Yang PB, Atkins KD, Dafny N. Behavioral sensitization and crosssensitization between methylphenidate amphetamine, and 3,4-methylenedioxymethamphetamine (MDMA) in female SD rats. Eur $J$ Pharmacol. 2011;66:72-85

22. Paxinos G, Watson C. The Rat Brain in Stereotaxic Coordinates. 2nd ed. London: Academic Press; 1986.

23. Chong SL, Claussen CM, Dafny N. Nucleus accumbens neuronal activity in freely behaving rats is modulated following acute and chronic methylphenidate administration. Brain Res Bull. 2012;10: 445-456.

24. Claussen CM, Dafny N. Acute and chronic methylphenidate modulates the neuronal activity of the caudate nucleus recorded from freely behaving rats. Brain Res Bull. 2012;87:387-396.

25. Dafny N. The hypothalamus exhibits electrophysiologic evidence for morphine tolerance and dependence. Exp Neurol. 1982;77:66-77.

26. Dafny N, Terkel J. Hypothalamic neuronal activity associated with onset of pseudopregnancy in the rat. Neuroendocrinology. 1990;51: 459-467.

27. Yang PB, Swann AC, Dafny N. Chronic methylphenidate modulates locomotor activity and sensory evoked responses in the VTA and NAc of freely behaving rats. Neuropharmacology. 2006;51:546-556.

28. Yang PB, Swann AC, Dafny N. Sensory-evoked potentials recordings from the ventral tegmental area, nucleus accumbens, prefrontal cortex, and caudate nucleus and locomotor activity are modulated in dose-response characteristics by methylphenidate. Brain Res. 2006;1073-1074:164-174.

29. Gaytan O, Ghelani D, Martin S, Swann A, Dafny N. Dose response characteristics of methylphenidate on different indices of rats' locomotor activity at the beginning of the dark cycle. Brain Res. 1996;727: 13-21.

30. Yang PB, Swann AC, Dafny N. Dose-response characteristics of methylphenidate on locomotor behavior and on sensory evoked potentials recorded from the VTA, NAc, and PFC in freely behaving rats. Behav Brain Funct. 2006;2:3.

31. Dafny N. Neurophysiological evidence for tolerance and dependence on opiates: simultaneous multiunit recordings from septum, thalamus, and caudate nucleus. J Neurosci Res. 1980;5:339-349.

32. Salek RL, Claussen CM, Perez A, Dafny N. Acute and chronic methylphenidate alters prefrontal cortex neuronal activity recorded from freely behaving rats. Eur J Pharmacol. 2012;679:60-67.

33. Dietz DM, Dietz KC, Nestler EJ, Russo SJ. Molecular mechanisms of psychostimulant-induced structural plasticity. Pharmacopsychiatry. 2009; 1:S69-S78. 
34. Gronier B. In vivo electrophysiological effects of methylphenidate in the prefrontal cortex: involvement of dopamine D1 and alpha 2 adrenergic receptors. Eur Neuropsychopharmacol. 2011;21:192-204.

35. Arnsten AF. Toward a new understanding of attention-deficit hyperactivity disorder pathophysiology: an important role for prefrontal cortex dysfunction. CNS Drugs. 2009;1:33-41.

36. Seamans JK, Yang CR. The principal features and mechanisms of dopamine modulation in the prefrontal cortex. Prog Neurobiol. 2004; 74:1-58.
37. Wang M, Ramos BP, Paspalas CD, et al. Alpha2 A-adrenoceptors strengthen working memory networks by inhibiting cAMP-HCN channel signaling in prefrontal cortex. Cell. 2007;129:397-410.

38. Volz TJ. Neuropharmacological mechanisms underlying the neuroprotective effects of methylphenidate. Curr Neuropharmacol. 2008;6: 379-385.

39. Andrews GD, Lavin A. Methylphenidate increases cortical excitability via activation of alpha-2 noradrenergic receptors. Neuropsychopharmacology. 2006;31:594-601.

\section{Publish your work in this journal}

The Journal of Experimental Pharmacology is an international, peerreviewed, open access journal publishing original research, reports, reviews and commentaries on all areas of laboratory and experimenta pharmacology. The manuscript management system is completely online and includes a very quick and fair peer-review system.
Visit http://www.dovepress.com/testimonials.php to read real quotes from published authors. 